\title{
Short and Long-Range Electron Transfer Compete to Determine Free-Charge Yield in Organic Semiconductors
}

\author{
Joshua M. Carr ${ }^{1}$, Taylor G. Allen², Bryon W. Larson², Iryna G. Davydenko³, Raghunath R. \\ Dasari $^{3,5}$, Stephen Barlow ${ }^{3,4,5}$, Seth R. Marder ${ }^{3,4,5,6}$, Obadiah G. Reid ${ }^{2,4, *}$, and Garry Rumbles ${ }^{2,4,5, *}$
}

Abstract: Understanding how Frenkel excitons efficiently split to form free-charges in low-dielectric constant organic semiconductors has proven challenging, with many different models proposed in recent years to explain this phenomenon. Here, we present evidence that a simple model invoking a modest amount of charge delocalization, a sum over the available microstates, and the Marcus rate constant for electron transfer can explain many seemingly contradictory phenomena reported in the literature. We use an electron-accepting fullerene host matrix dilutely sensitized with a series of electron donor molecules to test this hypothesis. The donor series enables us to tune the driving force for photoinduced electron transfer over a range of $0.7 \mathrm{eV}$, mapping out normal, optimal, and inverted regimes for free-charge generation efficiency, as measured by time-resolved microwave conductivity. However, the photoluminescence of the donor is rapidly quenched as the driving force increases, with no evidence for inverted behavior, nor the linear relationship between photoluminescence quenching and charge-generation efficiency one would expect in the absence of additional competing loss pathways. This behavior is self-consistently explained by competitive formation of bound charge-transfer states and long-range or delocalized free-charge states, where both rate constants are described by the Marcus rate equation. Moreover, the model predicts a suppression of the inverted regime for high-concentration blends and efficient ultrafast free-charge generation, 
providing a mechanistic explanation for why Marcus-inverted-behavior is rarely observed in device studies.

\section{Author Affiliations:}

1. University of Colorado Boulder, Materials Science \& Engineering Program, Boulder CO 80303

2. National Renewable Energy Laboratory, Chemistry and Nanoscience Center, Golden CO 80401

3. Georgia Institute of Technology, School of Chemistry and Biochemistry, Atlanta GA 30332

4. University of Colorado Boulder, Renewable and Sustainable Energy Institute, Boulder CO 80303

5. University of Colorado Boulder, Department of Chemistry, Boulder CO 80303

6. University of Colorado Boulder, Department of Chemical and Biological Engineering, Boulder CO 80303

\section{*Corresponding Author Addresses:}

\section{Obadiah.reid@colorado.edu}

Garry.rumbles@nrel.gov 


\section{Introduction:}

Simple electrostatic arguments suggest that it should not be possible to make an efficient photovoltaic device from a material with a dielectric constant between 3 and 4. Yet single-junction organic photovoltaic (OPV) cells have now exceeded $18 \%$ power conversion efficiency, and progress continues apace. ${ }^{1}$ Many models have been proposed to describe photoinduced electron transfer (PET) between photoexcited donor and acceptor species in solid-state organic systems ${ }^{2-10}$ but a fully self-consistent, experimentally verifiable model has proved elusive. In particular, the disconnect between the solution-phase molecular PET community and the OPV world remains large. The description of electron transfer provided by Rudolf Marcus is the unquestioned foundation of work in the former field, ${ }^{11-13}$ while in the latter it has often been ignored or even explicitly discarded. It has been argued, for instance, that the Marcus formulation breaks down in OPV materials because of the high density of electronic states participating in electron transfer when extended molecular aggregates, such as when fullerene crystallites are present ${ }^{14}$ We seek to test this idea, exploring the limits of the Marcus rate equation (1) in describing electron transfer in the presence of these extended molecular aggregates:

$$
k_{P E T}=\frac{2 \pi}{\hbar}\left|H_{D A}\right|^{2} \frac{1}{\sqrt{4 \pi \lambda k_{B} T}} \exp \left[-\frac{\left(\lambda+\Delta G_{P E T}\right)^{2}}{4 \lambda k_{B} T}\right]
$$

Here, $\Delta G_{P E T}$ is the Gibbs energy change of the PET reaction, $\lambda$ is the reorganization energy associated with the differing nuclear geometry of reactants and products, and $H_{D A}$ is the electronic coupling (orbital overlap) between the donor (D) and acceptor (A) statesThe distinctive prediction of this model is an optimal value of $k_{P E T}$ where $\Delta G=-\lambda$. For larger values of $\Delta G$ the reaction becomes too exergonic, and an "inverted region" is

predicted where $k_{P E T}$ decreases as $|\Delta G|$ increases. 
We have demonstrated this "inverted region" several times using free-charge carrier yield as a proxy for $k_{P E T}$, but usually in systems with modest charge carrier mobility, and in particular without large fullerene aggregates that might be expected to introduce a wide manifold of charge-transfer states that could lead to a weakening or absence of rate-constant / yield inversion. ${ }^{15-17}$ Here, we report charge yield and photoluminescence quenching (PLQ) as a function of the driving force for PET from a series of molecular donors at low concentration in a 6,6-phenyl $\mathrm{C}_{61}$-butyric acid methyl ester (PCBM) host matrix. Despite the fullerene host and its anticipated high density of states, we observe normal, optimal, and inverted regimes of free charge generation using time-resolved microwave conductivity (TRMC). However, PLQ experiments on the same samples reveal that the quenching efficiency rises much faster than the free charge yield, and quickly reaches $100 \%$ while the free-charge yield peaks at $\sim 60 \%$. Moreover, there is no "inverted" regime observed for the PLQ, which remains at $100 \%$ even for the largest driving force $(-0.69 \mathrm{eV})$ we were able to test.

These results can be self-consistently described by a Distributed Range Electron Transfer (DRET) model where localized charge-transfer (CT) states kinetically compete with free-charge (FC) states for the available exciton population. In each case, the rate-constant for electron transfer is described by the Marcus rate equation (eqn. 1), allowing for differing reorganization energies and driving forces for the $\mathrm{FC}$ and $\mathrm{CT}$ species. A sum over the rate constants for transfer to the available microstates for bound and free charge pairs over a wide range of distances allows FC states with larger electron-hole separation distances to compete effectively with shorter-range, more tightly bound, CT states when $\Delta G$ is near the optimum (ca. $-0.4 \mathrm{eV})$. This process is enabled by a moderately long-range electron transfer process, described by an exponentially decaying electronic coupling element $\left(H_{D A}\right)$ at greater distances. These results unify our understanding of electron transfer in both solution and solid-state systems, and the model allows us to make experimentally testable predictions concerning the sub-gap FC and CT state spectra, the temperature-dependence of 
free-charge generation, and the behavior of the high concentration donor/acceptor blends used in OPV devices. Ultimately we predict that the high density of states available in high concentration donor:acceptor blends does indeed suppress the Marcus inverted regime in OPV devices, but that the Marcus rate equation remains foundational to our understanding of electron transfer in these systems.

We first describe our experimental design and results, demonstrating the apparent discrepancy between photoluminescence quenching and free charge yield, as well as evidence for an inverted regime for free charge yield in our fullerene host. This is followed by a detailed description of the DRET model, the predictions it makes possible, and comparison with relevant literature.

\section{Results and Discussion:}

Testing eqn. 1 requires two things: (1) an experimental observable that is controlled by $k_{P E T}$, and (2) the ability to tune $\Delta G$ across a useful range. Figure 1 illustrates our approach. To satisfy the first criteria we choose to measure both photoluminescence quenching yield $\left(\phi_{P L Q}\right)$ and free-charge carrier yield $\left(\phi_{F C}\right)$ as this allows observation of both the reactants (excitons) and products (free charges) of the reaction. Each of these can be connected to $k_{P E T}$ via:

$$
\begin{aligned}
& \phi_{F C}=\frac{k_{P E T}}{k_{r+n r}+k_{P E T}} \\
& \phi_{P L Q}=1-\frac{k_{r+n r}}{k_{r+n r}+k_{P E T}}
\end{aligned}
$$

Where $k_{r+n r}$ is the fluorescence rate constant of the reactant exciton. We ensure that $k_{P E T}$ is the controlling rate constant by eliminating the possibility of exciton diffusion or energy transfer to a host-centered excited state; we use extreme dilution $\left(0.005 \mathrm{~mol} \mathrm{~kg}^{-1}\right)$ of electron-donating guest molecules in a solid solution of our electron acceptor (PCBM), and choose donors (phthalocyanines, naphthalocyanines, and squaraines, Figure 1e) that cannot transfer their exciton energy to PCBM, though energy transfer to weakly absorbing charge-transfer states cannot be entirely ruled out. Figure 1a quantitatively illustrates the 
low concentration of donors in our films, and $\mathbf{1 b}$ contrasts it with a 1:1 mole fraction film. Evidence for the isolation of our sensitizers comes from absorption spectroscopy (Figures 1c, S1). Figure 1c shows, as examples, absorption spectra of a PCBM film sensitized with a phthalocyanine guest donor (red), the neat PCBM (brown), and the phthalocyanine in polystyrene and chlorobenzene solution (green and black, respectively). The extensive red-shifting and broadening typical of aggregated phthalocyanines is absent in these films, suggesting that we have indeed prepared samples that resemble a solid solution - it does not resemble the typical aggregate spectra of pure phthalocyanine films. ${ }^{18-21}$

The symmetric broadening and slight red shift of phthalocyanine spectrum in the PCBM host may be due to a combination of effects. First, we note that this phenomenon is universal for all our sensitizers, even those (Sq1 and Sq2) that we will show below not to exhibit strong PL quenching or free-charge generation. Raising the refractive index of the medium (from $\sim 1.5-1.6$ in solution or polystyrene to $\sim 1.9-2$ in the PCBM host $^{22,23}$ ) would be expected to red-shift even modestly dipolar excited states, and PCBM doping has been shown to produce just this effect, even at low weight percentages. ${ }^{24}$ It is also possible that there are contributions from the often observed charge-transfer absorption at the donor acceptor interface. ${ }^{25-27}$ The latter effect is deemed to be an unlikely explanation, however, as CT-absorption is usually confined to states far down the absorption tail with oscillator strengths at least two-orders of magnitude less than the primary excited state, and we do not observe charge-transfer emission bands in any of our PL data, nor is there any systematic dependance of the red-shift or broadening on $\Delta G$, as would be expected. ${ }^{28}$ 
(a)

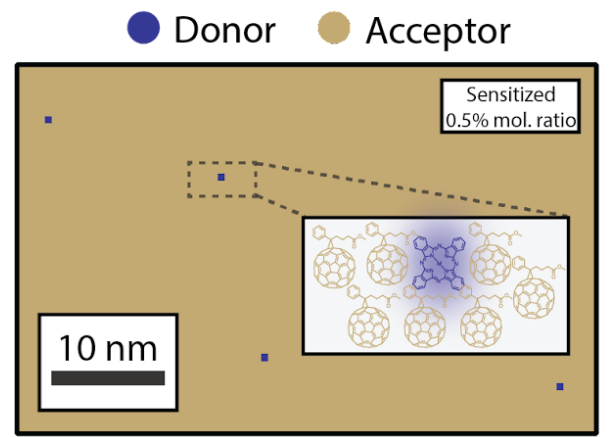

(c)

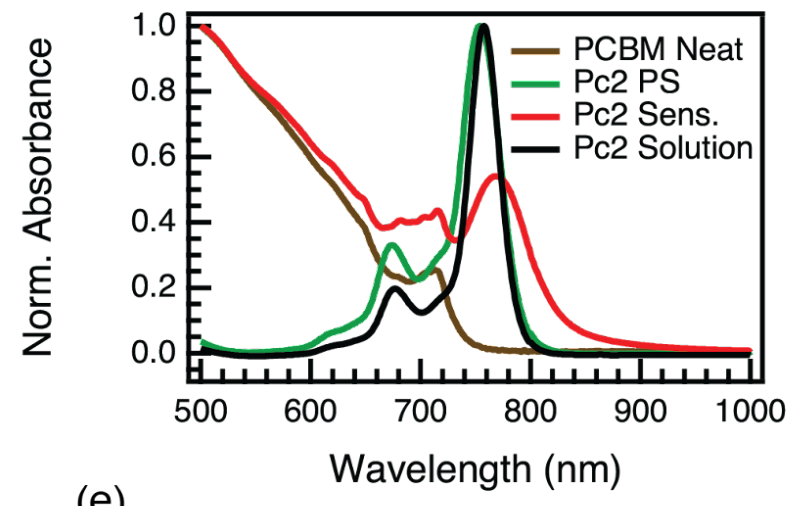

(b)

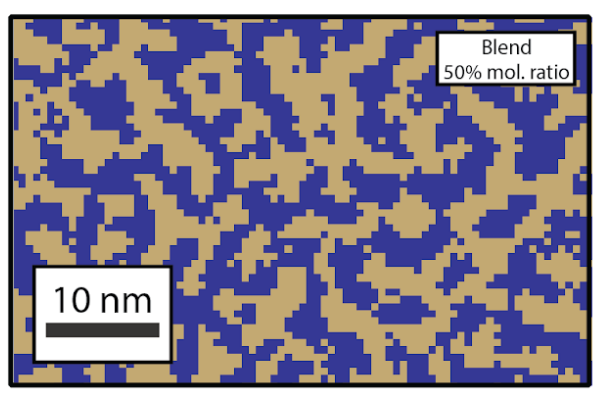

(d)

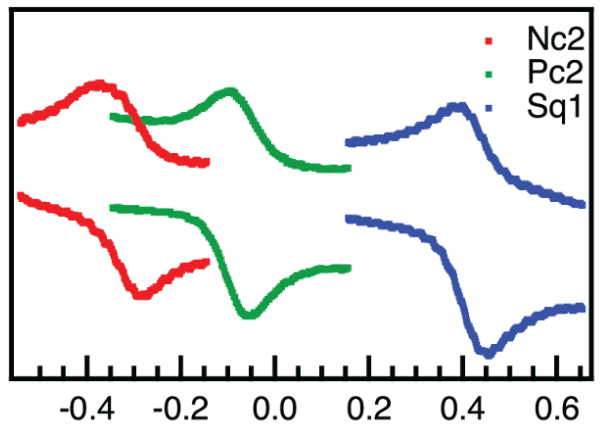

Potential vs. $\mathrm{Fc} / \mathrm{Fc}^{+}(\mathrm{V})$

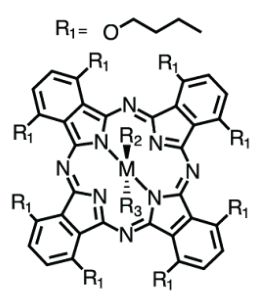

$\begin{array}{ll} & M \\ \text { Pc1 } & 2 \mathrm{H} \\ \text { Pc2 } & \mathrm{Si}\end{array}$

Pc3 Zn

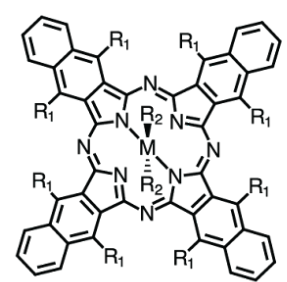

$\mathrm{B}_{1}$

$R_{1} \quad R_{2} R^{2}(n-h e x y) I_{3}$

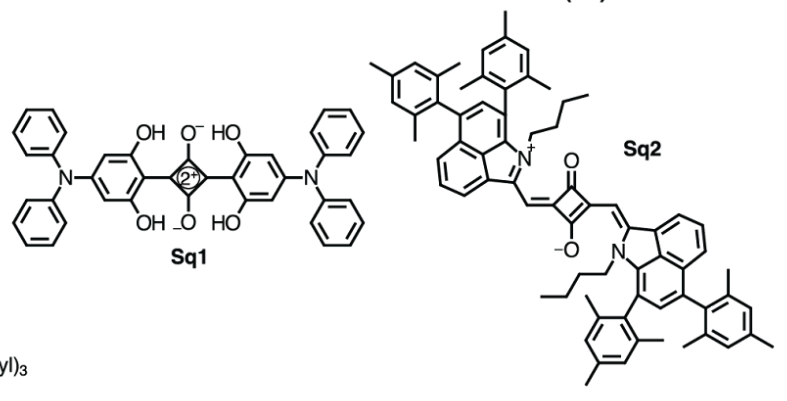

Figure 1. a. Quantitative illustration of a $0.5 \%$ mol-fraction sensitized film with a random distribution of donors. Inset image shows a $5 \mathrm{~nm}$ scale zoomed in description of the microstructure with isolated donors in the PCBM host. b. Illustration of a 50:50 mole fraction film. In both cases the tan color is used to represent PCBM while the blue is the sensitizer. c. Normalized absorption spectra of Pc2 in $1 \mu \mathrm{M}$ chlorobenzene solution (black), of Pc2 as sensitizer at $0.005 \mathrm{~mol} \mathrm{~kg}^{-1}$ in polystyrene (green), or PCBM (red), and of neat PCBM (brown) films. d. Cyclic voltammograms showing $\sim 1 \mathrm{~V}$ range in oxidation potential achieved using our series of donor molecules. e. Molecular sensitizer structures and abbreviations used in this work (see also Table 1). 
The second criteria above, tuning $\Delta G$, was accomplished by choosing a series of donor derivatives with widely varying reversible oxidation potentials (characterized using cyclic voltammetry, Figure 1d,e), which, along with a moderate variation in excited-state energies (Figure S2 and Table S1), afford a $\sim 0.7 \mathrm{eV}$ range in $\Delta G$ (Figure 1d,e, Table 1).

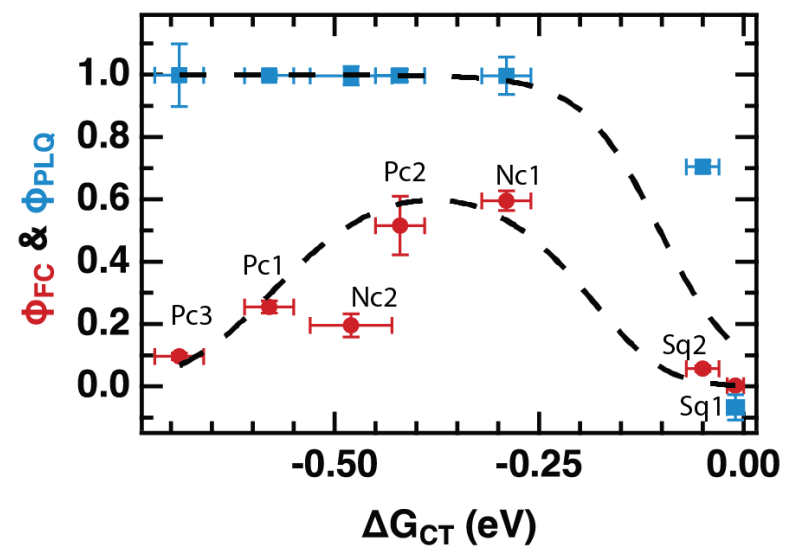

Figure 2. Photoinduced free electron yield $\left(\phi_{F C}\right.$, red markers) and PLQ yield ( $\phi_{P L Q}$ blue markers). Data points are labeled with their corresponding sensitizer (Figure 1e and Table 1). The dashed curves are the result of a global fit to both $\phi_{P L Q}$ and $\phi_{F C}$ using the DRET model, described by eqns. 1,6-12. Fit parameters are given in Table 2. Error bars are standard errors calculated from replicate measurements as described in the Experimental.

The primary experimental result of this work is shown in Figure 2, which displays $\phi_{F C}$ measured by TRMC and steady-state PLQ yield, $\phi_{P L Q}$, as a function of the nominal Gibbs energy for PET to a localized charge-transfer state, $\Delta G_{C T}$ (eqn. 5). Each data point represents one of the seven sensitizers (Figure 1e and Table 1) at $0.005 \mathrm{~mol} \mathrm{~kg}^{-1}$ in a host matrix of PCBM. A Marcus-like trend in $\phi_{F C}$ with increasing $\Delta G_{C T}$ is evident, with a distinct optimum of ca. $-0.4 \mathrm{eV}$ and a pronounced inverted regime at $\Delta G_{C T}<-0.4 \mathrm{eV}$. The $\Delta G_{C T}$-dependence of the $\phi_{P L Q}$, on the other hand, shows a rapid increase in quenching from ca. $0--0.3 \mathrm{eV}$, which then saturates at $\sim 100 \%$. In all cases, selective excitation of our red-absorbing sensitizers beyond the absorption onset of PCBM eliminates the possibility of exciton diffusion and/or energy-transfer processes. 
Ordinarily TRMC experiments do not directly provide the yield of free-charges, but rather the product of free-charge carrier yield and the sum of the electron and hole mobilities $(\phi \Sigma \mu)^{29,30}$

$$
\phi \sum_{i} \mu_{i}=\phi_{e} \mu_{e}+\phi_{h} \mu_{h}
$$

where $\phi_{e}$ and $\mu_{e}$ are the yield and mobility of electrons and $\phi_{h}$ and $\mu_{h}$ are the yield and mobility of holes, respectively. Two aspects of our experimental design allow us to assign the ordinate of Figure 2 quantitatively to $\phi_{e}$ (labeled as $\phi_{F C}$ for generality in our DRET model): (1) the use of dilute $\left(0.005 \mathrm{~mol} \mathrm{~kg}^{-1}\right)$ donor molecules in an electron accepting host (PCBM) eliminates the hole mobility contribution; and (2) the fact that the electron mobility at our $\sim 9 \mathrm{GHz}$ microwave probe frequency is known from prior work by both Warman et al. and Ferguson $\left(\mu_{e}=0.040-0.059 \mathrm{~cm}^{2} \mathrm{~V}^{-1} \mathrm{~s}^{-1}\right) .{ }^{31,32}$

The driving-force axis of Figure 2, $\Delta G_{C T}$, was created by choosing our series of donor molecules with appropriately varied oxidation potentials and exciton energies (Table 1) to act as guest sensitizers in the PCBM host (Figure 1d and 1e). We calculate $\Delta G_{C T}$ according to:

$$
\Delta G_{C T}=E_{o x, D}-E_{r e d, A}-E_{e x}
$$

where $E_{o x, \mathrm{D}}$ is the half-wave oxidation potential of the donor, $E_{r e d, \mathrm{~A}}$ is the half-wave reduction potential of the acceptor, and $E_{e x}$ is the energy of the lowest lying exciton in the system, all expressed in electron-volts. (See Fig. S2-S4 and discussion for details on these quantities). We use the subscript "CT" to indicate that this is a simplified version of the Gibbs energy change for PET. The full form is: 


$$
\Delta G_{P E T}=E_{o x, D}-E_{r e d, A}-E_{e x}+\Delta G_{S}+W\left(D^{+} / A^{-}\right)
$$

where the two additional terms in eqn. $\mathbf{6}$ are the Born correction, $\Delta G_{S}^{33}$ and the electrostatic work needed to form the product state, $W\left(D^{+} / A^{-}\right) \cdot{ }^{34}$ The former accounts for the difference in dielectric medium in which the redox potentials were measured versus that in which the PET reaction takes place; the latter accounts for the energy needed to separate the charges to their final distance. It turns out that if the dielectric constants for the $\mathrm{CV}$ measurements of $E_{o x, D}$ and $E_{r e d, A}$ are equal and much larger than that pertaining to the PET reaction $\left(\varepsilon_{A}=\varepsilon_{D}>>\varepsilon\right)$, and the smallest available charge transfer distance $\left(\mathrm{R}_{\mathrm{DA}}\right)$ is about equal to the donor and acceptor radii (i.e. $r_{D} \approx r_{A} \approx R_{D A}$ ) these two terms reduce to:

$$
\Delta G_{S}+W\left(D^{+} / A^{-}\right)=\frac{q^{2}}{4 \pi \varepsilon \varepsilon_{0}}\left(\frac{1}{r}-\frac{1}{r_{0}}\right)
$$

Here, $r_{0}$ is the initial radius of the reactant exciton, $r$ is the final separation in the product state, and $\varepsilon$ is the relative dielectric constant in which PET takes place. Evidently, if the charge-transfer product has a radius similar to the initial excited state $\left(r \approx r_{0}\right)$, eqn. 7 vanishes, reducing eqn. 6 back to eqn. 5 . Another way of interpreting this statement is that the most localized product species (CT state) has the same electrostatic binding energy as the reactant exciton. Thus our assignment of eqn. 5: it expresses the Gibbs energy change with respect to the nearest-neighbor CT state. In what follows we consistently use $\Delta G_{C T}$ to characterize the "driving force" for electron transfer, as it is a convenient quantity based on solid experimental data. However, eqns. 6 and 7 are ultimately vital in the full analysis of our data.

Table 1. Tabulated average $E_{o x, D}$ and $E_{e x}$ for all sensitizers and $\Delta G_{C T}$ for each sensitizer:PCBM pair using measured $E_{r e d, A}$ of $-1.07 \mathrm{~V}$ for PCBM. All redox potentials are vs. $\mathrm{Fc} / \mathrm{Fc}^{+}$. The error associated with the driving force is propagated from the averaged 
oxidation and reduction potentials. All CV scans are shown in Figure S4. $E_{e x}$ values are from spectra in Figure S2. *Assuming a one-electron redox reaction, these half-wave potentials can be expressed in units of $\mathrm{eV}$ instead of $\mathrm{V}$.

\begin{tabular}{cccc}
\hline Sensitizer & $\begin{array}{c}E_{e x}{ }^{*} \\
(\mathrm{eV})\end{array}$ & $\begin{array}{c}E_{\text {ox,D }}{ }^{*} \\
(\mathrm{eV})\end{array}$ & $\Delta G_{C T}(\mathrm{eV})$ \\
\hline $\mathbf{S q 1}$ & 1.64 & 0.56 & $-0.01 \pm 0.03$ \\
$\mathbf{S q 2}$ & 1.24 & 0.12 & $-0.05 \pm 0.05$ \\
$\mathbf{N c 1}$ & 1.58 & 0.22 & $-0.29 \pm 0.01$ \\
$\mathbf{P c 2}$ & 1.62 & 0.13 & $-0.42 \pm 0.03$ \\
$\mathbf{N c 2}$ & 1.39 & -0.16 & $-0.48 \pm 0.03$ \\
$\mathbf{P c 1}$ & 1.59 & -0.06 & $-0.58 \pm 0.03$ \\
$\mathbf{P c 3}$ & 1.60 & -0.16 & $-0.69 \pm 0.02$ \\
\hline
\end{tabular}

Taken by itself, the trend in $\phi_{F C}$ as a function of driving force in Figure 2 may be readily explained by the Marcus formulation for the rate constant of PET $\left(k_{P E T}\right.$, eqn. 1) if one makes the assumption that the only rate-constant in the system that changes as a function of driving force is $k_{P E T}$, as was done in eqn. 2 . However, the trend in $\phi_{P L Q}$ observed in Figure 2 appears to contradict this simple hypothesis. $\phi_{P L Q}$ increases rapidly with $\Delta G_{C T}$, and there is no evidence of an inverted regime. Furthermore, we observe a maximum $\phi_{F C}$ at the optimum (ca. $0.3-0.4 \mathrm{eV}$ ) of only $\sim 60 \%$, which is considerably lower than $\phi_{P L Q}$, and lower than one would expect assuming values of $k_{P E T} \sim 10^{12} \mathrm{~s}^{-1}$ typically reported in OPV materials and observed in our TA experiments (see SI Figures S7-15). We can mostly rule out the possibility that the low apparent yield of charges is simply due to an incorrect mobility value, as the $\phi_{F C}$ curve remains reasonably bell-shaped without the flat-top one would expect if the free-carrier yield approached $100 \%$ over any considerable range (see Figure $4 \mathbf{b}$ for an example). Similarly, the low free-charge yield and quenching efficiency cannot be accounted for by heterogeneity in the samples, where some sensitizers undergo ultrafast PET while others do not. Such a model would also predict a broad flat-topped shape in $\phi_{F C}$ vs. $\Delta G_{C T}$ and would not explain why $\phi_{P L Q}=100 \%$ across such a broad range. 
We posit that the conflict observed above between the TRMC, PL, and TA measurements may be most simply explained through a competition between free-charge (FC), and localized charge-transfer (CT) states, partitioning $k_{P E T}$ into two components: $k_{P E T}=k_{F C}+k_{C T}$, where CT states are a loss pathway, not an intermediate leading to FC. Figure 3a conceptually illustrates this kinetic scheme with both the electrostatic potential and the Gibbs energy curve accounting for configurational entropy of the continuum of FC and CT states plotted.

(a)

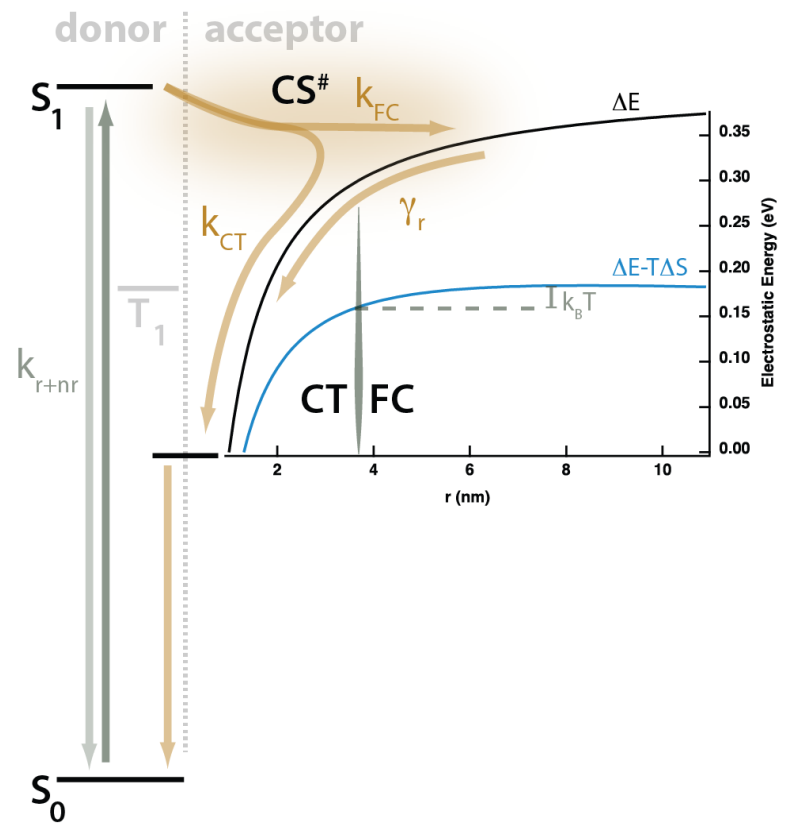

(b)

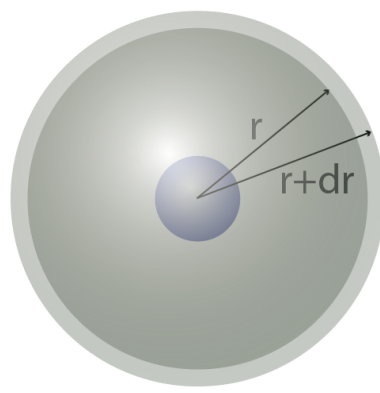

(c)

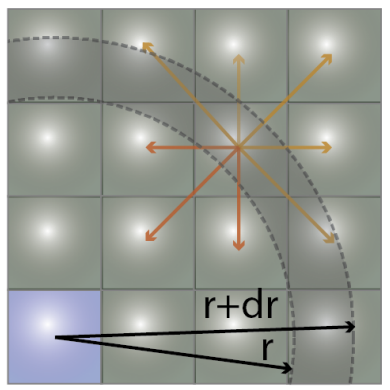

Figure 3. a. Proposed kinetic model showing competing processes of charge transfer to localized CT states vs. FC states. The black trace is the coulomb work term from eqn. $\mathbf{6}$ as a function of the separation distance between the electron and hole, $r$. The blue trace is a Gibbs energy curve including an entropic correction accounting for the number of sites available for charge transfer using eqn. 11. The distinction between $\mathrm{FC}$ and $\mathrm{CT}$ states is defined by the point at which the Gibbs energy curve is within $1 k_{B} T$ of its peak value, denoted $r_{c}$. The kinetic process is as follows: 1. Selective photoexcitation of the sensitizer (donor), 2. Exciton dissociation into either FC or CT states with rate constants $k_{F C}$ and $k_{C T}$, 3. ultimate recombination of FC states via the bimolecular rate-constant $Y_{r}$. Both the exciton and the CT states may decay directly to the ground-state. b. Illustration of the spherical shells of acceptor sites (grey) available to participate in electron transfer at any given radius, $r$, from the donor (blue); calculated according 
to eqn. 11. c. A microscopic cartoon depicting the influence of entropy after electron transfer, where there are more pathways that lead to separation (yellow) than those that lead to recombination or CT state formation (red).

Upon photoexcitation a singlet exciton $\left(\mathrm{S}_{1}\right)$ is formed on the donating sensitizer, and PET takes place across a wide distribution of distances, which we partition into FC states forming with the cumulative rate constant $k_{F C}$, or CT states forming with cumulative rate constant $k_{C T}$. The latter may decay to the ground state but do not dissociate to form free-charges due to their binding energy. Mobile charges in FC states may recombine with bimolecular rate constant $\left(\gamma_{r}\right)$ through the localized CT states. The proposal of a delocalized charge-separated transition state, $\mathrm{CS}^{\#}$, intermediate between the exciton and CT or FC states is not required by our present data, but it is consistent with the need for coupled donor or acceptor aggregates to produce free-charges. ${ }^{16,24-26}$ Whether this state is real or representative of a tunneling probability or wavefunction overlap between the donor and a distant acceptor is a matter of interpretation.

The kinetic scheme described above forms the basis of the analytical model with which we globally fit our $\phi_{P L Q}$ and $\phi_{F C}$ curves in Figure 2. We refer to it as the Distributed Range Electron Transfer (DRET) model, as its key characteristic is a wide distribution in the range of PET and kinetic competition between free and trapped states.

Our derivation begins by re-defining FC and PLQ yields as a function of the partitioned rate constants, $k_{F C}$ and $k_{C T}$ :

$$
\begin{aligned}
& \phi_{F C}=\frac{k_{F C}}{k_{r+n r}+k_{F C}+k_{C T}} \\
& \phi_{P L Q}=1-\frac{k_{r+n r}}{k_{r+n r}+k_{F C}+k_{C T}}
\end{aligned}
$$


As the distinction between FC and CT states is purely one of charge-separation distance $(r)$ within a continuous distribution, the full form of the Gibbs energy change for PET in eqn. 6-7 combined with the Marcus rate equation (eqn. 1) can be used to describe this competition quantitatively, as different separation distances imply a different Gibbs energy change, and thus different rate constants. All that remains is to define the critical radius at which we partition this continuous distribution of states into "FC" and "CT" species, $r_{c}$; a suitable probability distribution, $P(r)$, that describes the likelihood of an electron tunneling any given distance, $r$, from the donor; and a description of the number of microstates available for charge-transfer as a function of distance, $\Omega(r)$. The product of these components is integrated over $r$ in order to appropriately partition $k_{P E T}$ :

$$
\begin{aligned}
& k_{C T}=\int_{r_{0}}^{r_{c}} k_{P E T}(r) \Omega(r) P(r) d r \\
& k_{F C}=\int_{r_{c}}^{\infty} k_{P E T}(r) \Omega(r) P(r) d r
\end{aligned}
$$

Recent literature has extensively discussed the potential role of configurational entropy $(S=$ $\left.-k_{B} \ln [\Omega(r)]\right)^{8,9,35-37}$ disorder, $^{2,6,9}$ and charge-transfer state delocalization ${ }^{2,38-40}$ (related to $P(r)$ ) in understanding charge separation in OPV materials. These studies guide our choice of $r_{c}$, the form of $\Omega(r)$, and that of $P(r)$, drawing particular inspiration from the work of Ratner, ${ }^{11}$ Gregg, ${ }^{8}$ Kassal. ${ }^{9}$ and Troisi. ${ }^{7}$

Our experiments yield a particularly simple form for $\Omega(r)$ : isolated donor molecules in an electron accepting host. The hole is fixed in space on the isolated donor, and a spherical shell of acceptor molecules is available to accept an electron at any given radius as illustrated in Figure 3b, thus:

$$
\Omega(r)=\frac{4}{3} \pi \frac{\left((r+a)^{3}-r^{3}\right)}{a^{3}} \xi
$$


Where $a=1 \mathrm{~nm}$ is the diameter of each site available to accept a charge and $\xi$ is the maximum possible packing efficiency of spheres (0.74). Numerically, a floor function is applied such that (eqn. 11) will always provide a conservative integer number of available sites. This equation allows us to calculate the entropic contribution to the energy surface for charge separation, and predict spontaneity, as shown in Figure 3a assuming $T=300 \mathrm{~K}$ (blue trace). We choose the critical radius, $r_{c}$, to be the point within $1 k_{B} T$ of the peak of this Gibbs energy curve, which comes out to be $r_{c}=3.88 \mathrm{~nm}$ under these conditions. This number corresponds closely both with experimentally determined initial electron-hole distance distributions in efficient OPV materials, ${ }^{41,42}$ and with the initial CT-distance needed to avoid geminate recombination in $3 \mathrm{D}$ Monte Carlo models ${ }^{3}$ in the absence of large disorder or delocalization effects. ${ }^{2}$ Notably, our choice of $r_{c}$ as being slightly below the peak of the Gibbs energy curve (blue) in Figure 3a is an implicit acknowledgement that a modest amount of disorder and/or delocalization will exist in our samples, and is likely to aid in dissociation of charges, ${ }^{2,9}$ though we do not include either explicitly in our model. Finally, we define $P(r)$ as a peak-normalized exponential distribution, in accordance with long observed trends in distance-dependence of electron transfer: ${ }^{7,11}$

$$
P(r) d r=\left\{\begin{array}{l}
r \leq r_{0}: 1 \\
r>r_{0}: e^{-\beta\left(r-r_{0}\right)}
\end{array}\right\}
$$

where $\beta$ is the attenuation constant for long-range ET, and $r_{0}$ is as previously defined.

Eqns. 1,6-12 constitute a complete analytical model with which we globally fit our $\phi_{P L Q}$ and $\phi_{F C}$ data in Figure 2 (global fit procedure in the SI). The resulting fit parameters along with associated uncertainties from the orthogonal distance regression we used are shown in Table 2. The only free fit parameters are $\beta, \lambda_{F C}$ , and $\lambda_{C T}$, and $H_{D A}$. The fluorescence rate constant $\left(k_{r+n r}\right)$ was held constant at the average value we obtained from time-resolved PL measurements (Figure S16) conducted on each sensitizer; $r_{C}$ was assigned as $3.88 \mathrm{~nm}$ as described above. 
Table 2. Parameter values from global fit of $\phi_{F C}$ and $\phi_{P L Q}$ data using the DRET model and fits shown in Figure 2. Reported errors associated with each parameter value are the standard deviation of the mean from the orthogonal distance regression procedure used to consider the standard errors in driving force, yield, and PLQ equally. Standard errors in this manuscript are described in Experimental.

\begin{tabular}{cc}
\hline Fit parameter & Value \\
\hline$\lambda_{F C}(\mathbf{e V})$ & $0.25 \pm 0.06$ \\
$\lambda_{C T}(\mathbf{e V})$ & $0.29 \pm 0.2$ \\
$\beta\left(\AA^{-1}\right)$ & $7.3 \times 10^{8}$ (held) \\
$k_{r+n r}\left(\mathbf{s}^{-1}\right)$ & $2.3 \pm 6$ \\
$H_{D A}(\mathbf{m e V})$ & 3.88 (held) \\
$r_{C}(\mathbf{n m})$ &
\end{tabular}

The fits we obtain to $\phi_{F C}$, and $\phi_{P L Q}$ are both quite good. In particular, the DRET model successfully captures the divergence between $\phi_{P L Q}$ and $\phi_{F C}$ observed in Figure 2. A key feature that makes this possible is that we allow the FC and CT states to take on different reorganization energies. While there is no quantitative physical basis for this assumption, we suggest that it may be connected with localization of these states. The far more localized CT states present a larger, more polarizing electric field to the local environment. Similarly, a more localized anion will experience greater intra-molecular bond distortions than one that shares the electron among several molecules. Both of these effects may contribute to the differing reorganization energies that the model allows, though we note that having artificially partitioned a continuum of states into the binary pair "FC" and "CT", these reorganization energies can only represent the average value associated with these categories. Strikingly, the values of $\beta$ and $H_{D A}$ that emerge from the fit are exactly in the range predicted by Troisi, ${ }^{7}$ and the former is well within previous experimental measurements for conjugated "bridge" molecules. ${ }^{43}$

We tested the unique ability of the DRET model to explain our data by comparing it to an equivalent formulation that treats nearest-neighbor CT states as the intermediate between the exciton and the FC states. 
This sequential model cannot fit our data well. In particular, such a model cannot reproduce the divergence between PL quenching and free charge yield (see Figure S19). However, it must be admitted that just the right systematic error in our charge yield measurements in just the right direction could lead to an adequate fit using both models. In addition, alternative interpretations to the long-range PET we observe may exist, including energy transfer from the exciton to an optically-active band of CT and FC states commonly observed in similar donor:acceptor blends. ${ }^{25-27}$ Another possible explanation for the divergence between $\phi_{P L Q}$ and $\phi_{F C}$ could be a fast internal conversion pathway that competes with PET via partial-CT states, which may exist in sensitized PCBM films but not for isolated donor molecules in the inert polystyrene matrix used for our control samples. At present, however, the DRET model remains the simplest and most self-consistent way to explain our data, and only future experiments testing its predictions will serve to distinguish these competing possibilities with greater certainty. 

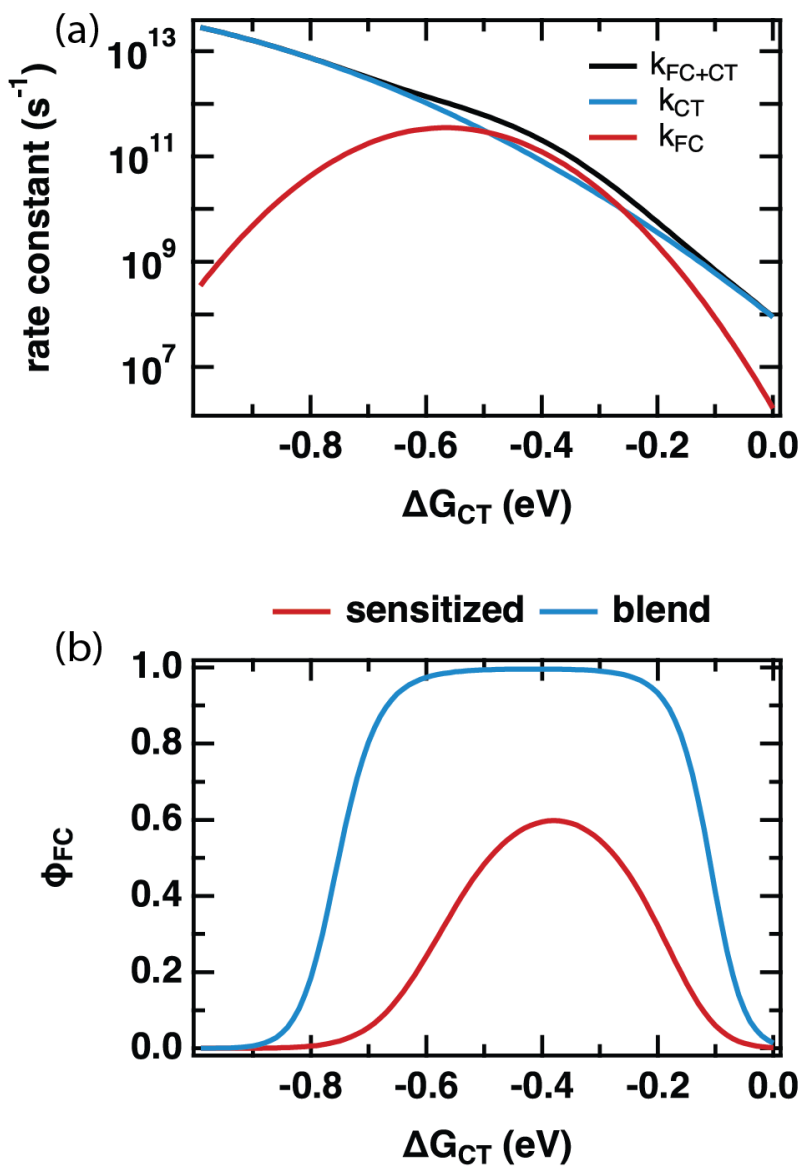

Figure 4. a. PET rate constant as a function of $\Delta G_{C T}$ as is predicted by the DRET model (eqns. 1,6-12) using the fit parameters in Table 2. b. FC yield $\left(\phi_{F C}\right)$ as a function of $\Delta G_{C T}$ for the sensitized system studied in this work (red) and that of a predicted blend system as in Hood et al. (blue), which modifies the entropy through an increase in the number of available sites due to large aggregates of both donor and acceptor, rather than an isolated donor molecule in an accepting host. ${ }^{27}$

Comparing the values of $k_{C T}$ and $k_{F C}$ predicted by the DRET model as a function of $\Delta G_{C T}$ provides insight into how it is able to reproduce our experimental observations in Figure 2. Figure 4a shows these individual rate constants calculated as a function of $\Delta G_{C T}$ for the same fit parameters as in Table 2. Here, $k_{F C}$ is observed to be sharply peaked while $k_{C T}$ continues to increase across this range of $\Delta G_{C T}$ due to the difference in reorganization energy of the two species ( $0.25 \mathrm{vs} 1.3 \mathrm{eV}$, respectively). Thus, the observed 
"inverted region" in our FC yield, and the lack of one in the PLQ yield is explained: the high reorganization energy for the CT state, combined with the distance-dependence of $\Delta G_{P E T}$ in eqn. 6, allows $k_{C T}$ to out-compete $k_{F C}$ at low driving force and at high driving force. Only in the intermediate regime does $k_{F C}$ win out, leading to a maximum FC yield around $-0.4 \mathrm{eV}$ despite a reorganization energy of $0.25 \mathrm{eV}$. Notably, the overall rate constant for charge transfer (black curve) is ultrafast, as we observe in TA, and consistent with the literature. For any value of $\Delta G_{C T}$ exceeding $-0.3 \mathrm{eV}$ the rate constant is $10^{11} \mathrm{~s}^{-1}$ or greater and exceeds $10^{12} \mathrm{~s}^{-1}$ for the -0.6 $\mathrm{eV}$ driving force typical of the polymer:fullerene solar cells where subpicosecond charge separation is nearly universally observed. However, we do not attribute great significance to the absolute values of these rate constants, as they scale with the square of $H_{D A}$, the value of which is pinned in our experiments by just a few data points in the onset of the PL quenching curve.

Up to this point, we have shown that a relatively simple model based on the Marcus rate equation (eqn. 1) can explain our experimental data if localized CT states compete with free-charge generation rather than being the intermediate between excitons and free charges, as is most often assumed. Here, we qualitatively reconcile these results with a broad spectrum of literature, showing that our model predictions are consistent with previous observations, not just our own experimental data.

The first key question: why do TRMC and other experiments on sensitized films so frequently result in inverted free-carrier yields and relatively low peak yields, ${ }^{15-17,44}$ whilst inverted behavior has only rarely been observed in organic photovoltaic devices, ${ }^{45-47}$ and free charge yield is often close to $100 \%$ ? We posit that the difference lies in the number of microstates available to charge-separated species in high-concentration blends. Hood et al. have pointed out that the number of microstates is much higher for a quasi-planar interface between aggregated donors and acceptors than for an isolated donor in an accepting host. ${ }^{9}$ Figure $4 \mathbf{b}$ shows a comparison between our model, and an implementation that substitutes Hood's formulation for the number of microstates available in a high-concentration blend. In each case we use the 
same model parameters as in Table 2 but applied to these two different microenvironments. The red trace is the FC yield from an isolated donor in a homogenous mixture of acceptors, identical to our sensitized PCBM films, while the blue trace is for a planar interface between aggregated donors and acceptors. In the sensitized environment, the peak yield is $\sim 60 \%$ and includes both a slow turn-on in the normal region and slow turn-off in the inverted region, just as in our data. However, in the blend environment, the free-charge yield approaches $100 \%$, producing a wide flat peak with rapid turn-on and turn-off in the normal and inverted regions. The latter does not begin in earnest until $\Delta G_{C T}<-0.7 \mathrm{eV}$. We speculate that this behavior makes observation of an inverted region substantially more difficult in device studies, as much larger driving forces are required before it becomes evident. This is qualitatively consistent with the observations of Nakano et al., where a flat-topped curve was observed in photovoltaic devices and extremely exergonic reactions were required to observe inverted behavior. ${ }^{45}$

The second question concerns the nature and origin of the sub-gap "CT-state spectra" that have been widely observed in OPV materials. ${ }^{25-27}$ These data form the foundation from which localized CT-states were assigned as the intermediate between excitons and free charges, and have been broadly used as a method of characterizing the driving-force for PET. ${ }^{28,48}$ Intriguingly, very similar "CT-state" spectra are predicted by our DRET model. Given that $H_{D A}$ is an orbital overlap integral between the donor and the acceptor and $P(r)$ describes its attenuation as a function of distance, it seems reasonable to use these to estimate the relative oscillator strength of CT states as a function of distance (where "CT" here is used broadly to encompass all charge transfer distances), noting that optically-excited long-range electron transfer is known to occur, ${ }^{49}$ and much more sophisticated calculations have already predicted its potential importance in OPV materials. ${ }^{50}$ Combined with the number of microstates, $\Omega(r)$, and the energy of those states with respect to the initial exciton, $\Delta G_{P E T}(r)$, it is possible to calculate CT-state spectra for both isolated donors and planar interfaces using our DRET model, as shown in Figure S18. In the latter case the vast majority of these states have radii 
exceeding $r_{c}$, and can thus be characterized as FC states that would give rise to exactly the same sort of efficient sub-gap carrier generation that is commonly observed..$^{27,51}$

Finally, we address the temperature-dependence of free-charge generation. An attentive reader will have noted that our model is likely to predict a strong temperature dependence: $k_{P E T}$, and more importantly the value of $r_{c}$, are both explicitly temperature-dependent. This turns out to be true for isolated donors in an accepting host (Figure S17a). However, the DRET model predicts that for blends the temperature-dependence of free-charge generation will actually be quite weak down to $\sim 150 \mathrm{~K}$, consistent with previous experiments. ${ }^{52,53}$ At lower temperatures, however, the free-charge yield is predicted to decline precipitously (Figure S17b). This may be due in part to our use of the simplest form of the Marcus rate equation, without the quantum-mechanical corrections that capture the existence of zero-point vibrational energy and tunneling through the barrier. ${ }^{11}$

\section{Conclusions:}

We have demonstrated that the yield of free charges from PET in electron donor-sensitized PCBM films is dependent upon the driving force, exhibiting clear inverted behavior even in the presence of extended fullerene aggregates - consistent with an electron-transfer model based on the Marcus rate equation for PET. However, excitations are quenched much more efficiently than would be expected based on the free-charge yield with no complementary inverted region. These results are self-consistently explained by a Distributed Range Electron Transfer (DRET) model that describes free-charge generation as a competition between the formation of short-range charge-transfer states and long-range free-charge states, each separately described using the Marcus rate equation. An equivalent model that employs the nearest-neighbor CT states as the intermediate between excitons and free charges cannot fit our data. Moreover, the model accurately predicts the electron-transfer behavior of both dilutely sensitized and device-relevant concentration regimes through a 
simple change in how the number of available microstates for charge transfer is calculated, including the existence of sub-gap CT-state spectra and the temperature-dependence of free charge generation. Future work will explore this model in more detail, both the temperature dependence it predicts, and the donor concentration-dependence. These observations suggest a future unification of electron transfer theory in solution and solid-phase systems that will materially aid the advancement of science and technology based on electron-transfer reactions in molecular systems.

\section{Experimental Methods:}

Film Fabrication: Phenyl $\mathrm{C}_{61}$ butyric acid methyl ester (PCBM) was acquired from Nano-C at $99.9 \%$ purity and used as received. 1,4,8,11,15,18,22,25-octabutoxy-29H,31H-phthalocyanine (Pc1), zinc $1,4,8,11,15,18,22,25$-octabutoxy phthalocyanine (Pc3), 5,9,14,18,23,27,32,36-octabutoxy-2,3-naphthalocyanine and 2,4-bis[4-(N,N-diphenylamino)-2,6-dihydroxyphenyl] cyclobutene-diylium-1,3-bis(olate) (Sq1) were all acquired from Sigma-Aldrich at $>95 \%$ purity and used as received. Silicon 1,4,8,11,15,18,22,25-octabutoxyphthalocyanine triethylsiloxide hydroxide (Pc2) is from ref. $\left[{ }^{40}\right]$ and used as synthesized. Silicon 2,3-naphthalocyanine bis(trihexylsilyloxide) (Nc2) was acquired from Alfa Chemistry at $98 \%$ purity and used as acquired. 2,4-bis(1-butyl-6,8-dimesitylbenzo[cd]indol-2(1H)-ylidene)methyl)cyclobutene-diylium-1,3-bis(olate) (Sq2) was synthesized for this study and used as synthesized. This synthesis is given in the Supporting

\section{Information.}

Sample films were fabricated by ultrasonic spray-coating host-sensitizer solutions onto $25 \times 11 \mathrm{~mm}^{2}$ quartz substrates cleaned with acetone sonication for $10 \mathrm{~min}$ and $10 \mathrm{~min}$ of UV-ozone treatment. Stock solutions were prepared by dissolving each sensitizer in chlorobenzene at $1 \mathrm{mg} / \mathrm{mL}$, except for Pc3 which 
was dissolved in pyridine at $1 \mathrm{mg} / \mathrm{mL}$. PCBM and PS solutions were dissolved in chlorobenzene at 30 $\mathrm{mg} / \mathrm{mL}$. Host-sensitizer solution mixtures were made by mixing sensitizer solution with PCBM or PS host solution at $0.005 \mathrm{~mol} \mathrm{~kg}^{-1}$ for a total volume of $1 \mathrm{~mL}$. All films were spray coated in a nitrogen glovebox $(<1$ ppm $\mathrm{O}_{2}$ ). Spraying was accomplished by rastering the sample stage beneath the ultrasonic spray nozzle to coat a $50 \times 60 \mathrm{~mm}^{2}$ area containing three $25 \times 11 \mathrm{~mm}^{2}$ quartz substrates for making samples in triplicate under the same conditions. Atomized solution was delivered to the sample at a rate of $0.4 \mathrm{~mL} / \mathrm{min}$ using a syringe pump and air-shaping was applied with a $6 \mathrm{~L} / \mathrm{min}$ nitrogen stream to achieve fan-like jets for uniform spraying. The sample stage was heated to $100{ }^{\circ} \mathrm{C}$ to facilitate evaporation of high boiling solvents. Nozzle to substrate height was ca. $50 \mathrm{~mm} .5$ coats (repetitions of the raster routine) were done to achieve films ca. $1 \mu \mathrm{m}$ in thickness. PS and PCBM host films are made from the same spray coating parameters.

\begin{abstract}
Absorption Measurements: Optical absorption is characterized using a Varian Cary 5000 UV-Visible spectrophotometer with the Diffuse Reflectance Accessory (DRA) and an angled center mount. Spectra are collected in the transmittance configuration, but because we collect with the center mount in the DRA, it is effectively a transreflectance $(\% \mathrm{TR})$ spectrum, as both the reflectance $(\% \mathrm{R})$ and transmittance $(\% \mathrm{~T})$ are collected simultaneously. Excitation of the sample is with the full beam size which is centered on the film at an angle of incidence at $20^{\circ}$. The resolution of the instrument is $1 \mathrm{~nm}$ with grating changeovers at $800 \mathrm{~nm}$ and $350 \mathrm{~nm}$. A baseline is collected by inserting a blank, cleaned quartz substrate into the center mount of the DRA under the same collection settings. Both a $100 \%$ transreflectance and a $0 \%$ transflectance, where the beam is blocked, is collected to baseline the instrument before collection. Absorptance $(\% \mathrm{~A})$ is then calculated from the resulting spectrum by $\% \mathrm{~A}=100 \%-\% \mathrm{TR}$.
\end{abstract}

Photoluminescence spectroscopy and PL quenching: Photoluminescence spectra were collected using a custom-built Princeton Instruments spectrometer. A liquid nitrogen-cooled, front-illuminated Si CCD 
(PyLoN) was used for collecting visible-NIR spectra $(425-900 \mathrm{~nm})$ and a 1D liquid-nitrogen cooled InGaAs array (PyLoN-IR) was used for SWIR measurements (850-1550 nm). Vis-NIR spectra were intensity calibrated using an IntelliCal USB-LSVN (9000-410) calibration lamp. SWIR spectra were calibrated using a SWIR quartz tungsten halogen lamp from Princeton Instruments. Dual monochromators (HRS 500) were used to achieve pseudo-monochromatic excitation from an Energetiq EQ99x laser driven light source, with typical FWHM bandwidths $\sim 16 \mathrm{~nm}$ using a $1200 \mathrm{~g} / \mathrm{mm}, 750 \mathrm{~nm}$ blaze grating. A single monochromator was used for detection (Princeton HRS-300) with $1200 \mathrm{~g} / \mathrm{mm}$ (500 nm blaze) and $150 \mathrm{~g} / \mathrm{mm}$ (800 nm blaze) gratings used for measuring vis-NIR and SWIR spectra, respectively. Typical exposures were 0.5-1 s with 0.25-1 mm detection slit widths. PL spectra for each sensitizer:PS film were excited at $380 \mathrm{~nm}$. PL quenching experiments were accomplished by measuring PL of selectively excited sensitizers in inert polystyrene control samples and PCBM under identical conditions. PL quenching experiments were conducted by exciting the sensitizer:PCBM films at the same wavelengths used in the TRMC/TA experiments as denoted in Figure S1. Three spots were measured on each film to obtain an average PL spectrum such that an average quenching ratio can be calculated. As shown in eqn. S1, the integrated counts for each spectrum were normalized by $\% \mathrm{~A}$ at the excitation wavelength $\left(\lambda_{e x}\right)$ and the ratio of $\% \mathrm{~A}$-normalized, integrated counts between sensitizer:PS and sensitizer:PCBM films was used to calculate quenching. In practice, counts were divided by the power absorbed (counts/W absorbed) to account for power fluctuations.

$$
P L Q \text { ratio }=1-\left(P L_{P C B M} / A_{P C B M}\right) /\left(P L_{P S} / A_{P S}\right)
$$

Cyclic Voltammetry (CV): CV measurements were done in triplicate for each sensitizer and the PCBM against the $\mathrm{Fc} / \mathrm{Fc}^{+}$standard reference in an inert glovebox environment $\left(<1 \mathrm{ppm} \mathrm{O}_{2}\right)$. Experiments were performed on solutions of the sensitizer and PCBM in a 4:1 v/v ratio of dichlorobenzene to acetonitrile (Sigma-Aldrich 99.9\% anhydrous grade) with $0.1 \mathrm{M} \mathrm{Bu}_{4} \mathrm{~N}^{+} \mathrm{PF}_{6}^{-}($Sigma-Aldrich $>99 \%$ electrochemical grade) 
in order to make sure that both the electrolyte and the analyte were dissolved entirely. Electrochemistry Power Suite software was used to control equipment and execute scans. Three cyclic scans were done prior to each cyclic voltammogram collection to ensure analyte equilibration with electrode surfaces. Scan rates varied from 100-200 mV/s and each solution was scanned in both directions to ensure symmetry and reversibility. A "compact voltammetry cell research kit" (Pine product \# AKSPEKIT) was used to ensure the best repeatability of electrode placement from sample to sample. The cell includes a screen-printed three electrode system with a $2 \mathrm{~mm}$ Pt working electrode, a Pt counter electrode, and a silver wire pseudo-reference electrode. The electrodes and silver wire are rinsed and sanded between each measurement to prevent any contamination. Following scans, the $E_{1 / 2}$ of the first oxidation potential for the sensitizer is used to approximate the energy level of the donor and the $\mathrm{E}_{1 / 2}$ of the first reduction for the PCBM is used to approximate the energy level of the acceptor, both with reference to the $\mathrm{Fc} / \mathrm{Fc}^{+}$standard $\mathrm{E}_{1 / 2}$. This procedure is inspired by work from Larson et al. ${ }^{41}$ While CV measurements are not entirely indicative of the solid-state energetics for these sensitizers, we argue that the solution-like nature of the sensitization for the Pcs/Ncs/Sqs combined with the self-consistent dataset that we are able to achieve through $\mathrm{CV}$, that the relative shifts in the solid-state will be comparable so long as there is no aggregation. Furthermore, work done from Cardona et al. found that the conversion methods used for solution to solid-state energetics can vary greatly and use of a self-contained data set is more consistent. ${ }^{42}$ Errors for the CV measurements are determined from the standard deviation of the mean for the averaged triplicate solutions per sensitizer. Typical errors in this work are between $\pm 10-50 \mathrm{mV}$ for both the sensitizers and the PCBM.

TRMC Measurements: The TRMC technique has been described in detail in previous publications both in terms of the theory and the experimental setup. ${ }^{29,30}$ Film photoconductivity for this work is determined by the following: (1) TRMC transients are collected as a function of light intensity for each sample in the series to ensure that the response is linearly correlated. (2) Transients are fit with biexponential functions convoluted 
with the 7 ns cavity response. (3) The resulting peak value is normalized by the fraction of absorbed photons in the film. A Spectra-Physics PremiScan ULD/500 optical parametric oscillator pumped by a Spectra-Physics Quanta-Ray Nd:YAG laser was used to excite the samples with ca. 5 ns pulses in the peak absorption for each sensitizer as shown inset on the absorption figures in the SI Figure 1. TRMC transients with fits for each sample are shown in the SI Figure 5. TRMC measurement error is dominated by the error in measuring film absorption and errors associated with sample inconsistencies. The error shown for the yield data in Figure 2 is estimated by taking an average yield for three replicate films for each sensitizer, then taking the standard deviation of the mean.

Time-resolved photoluminescence (TRPL): Optical excitation with $\sim 100$ ps pulses at $700 \mathrm{~nm}$ was supplied by a NKT supercontinuum fiber laser (SuperK EXU-6-PP) with $2.69 \mathrm{MHz}$ repetition rate. A $10 \mathrm{~nm}$ bandpass filter was used to reduce the spectral bandwidth of the excitation beam. A Hamamatsu $300-900 \mathrm{~nm}$ (C10910-04) streak camera was used to collect time-resolved PL spectra. Instrument response was captured by scattering some excitation light into the detector using ground glass in the sample position. Transients were analyzed at the wavelength of maximum PL intensity for each film.

Standard Errors: Standard errors reported in this paper are from averaged repeated measurements from each experiment. In doing so, we report the averaged value and the standard deviation of the mean from the repeated measurements as the experimental value and the error for those experiments. If a quantity is determined from multiple experimental values, such as $\Delta G_{C T}$, then the error reported is the propagated error from each experimental value, combined in quadrature.

\section{Associated Content:}

Supporting Information: Absorption and emission characteristics of all samples and control samples, 
cyclic voltammograms for all sensitizer and host molecules, microwave conductivity transients for all samples and parameters from the global fits to each data set, femtosecond transient-absorption characterization of every sensitizer molecule including estimated triplet yields and ISC rate-constants, time-resolved photoluminescence characterization of every sensitizer molecule, temperature-dependance of FC yield and CT state spectra calculated using the DRET model, comparison of the DRET model with a sequential intermediate CT-state model, synthesis and characterization of the squaraine donors.

\section{Conflicts of Interest:}

The authors declare that there are no existing conflicts of interest associated with this content.

\section{Acknowledgements:}

This research was funded by the Solar Photochemistry Program, Division of Chemical Sciences, Geosciences, and Biosciences, Office of Basic Energy Sciences, U.S. Department of Energy (DOE). NREL is supported by the DOE under Contract No. DE-AC36-08GO28308. The U.S. Government retains (and the publisher, by accepting the article for publication, acknowledges that the U.S. Government retains) a nonexclusive, paid up, irrevocable, worldwide license to publish or reproduce the published form of this work, or allow others to do so, for U.S. Government purposes. Work at Georgia Tech and the University of Colorado-Boulder on squaraine synthesis and characterization was supported by the Office of Naval Research (N00014-21-1-2087 and N00014-21-1-2180).

\section{References:}

(1) Cui, Y.; Yao, H.; Zhang, J.; Xian, K.; Zhang, T.; Hong, L.; Wang, Y.; Xu, Y.; Ma, K.; An, C.; He, C.; Wei, Z.; Gao, F.; Hou, J. Single-Junction Organic Photovoltaic Cells with Approaching 18\% Efficiency. Advanced Materials 2020, 32 (19), 1908205. https://doi.org/10.1002/adma.201908205.

(2) Athanasopoulos, S.; Bassler, H.; Köhler, A. Disorder vs. Delocalization: Which Is More Advantageous for High-Efficiency Organic Solar Cells? The Journal of Physical Chemistry Letters 2019, 10 (22), 7107-7112. https://doi.org/10.1021/acs.jpclett.9b02866. 
(3) Peumans, P.; Forrest, S. R. Separation of Geminate Charge-Pairs at Donor-Acceptor Interfaces in Disordered Solids. Chemical Physics Letters 2004, 398 (1-3), 27-31.

https://doi.org/10.1016/j.cplett.2004.09.030.

(4) Yost, S. R.; Van Voorhis, T. Electrostatic Effects at Organic Semiconductor Interfaces: A Mechanism for "Cold" Exciton Breakup. The Journal of Physical Chemistry C 2013, 117 (11), 5617-5625. https://doi.org/10.1021/jp3125186.

(5) Burke, T. M.; McGehee, M. D. How High Local Charge Carrier Mobility and an Energy Cascade in a Three-Phase Bulk Heterojunction Enable $>90 \%$ Quantum Efficiency. Advanced materials (Deerfield Beach, Fla.) 2013, 26 (12), 1923-1928. https://doi.org/10.1002/adma.201304241.

(6) Eersel, H. van; Janssen, R. A. J.; Kemerink, M. Mechanism for Efficient Photoinduced Charge Separation at Disordered Organic Heterointerfaces. Advanced Functional Materials 2012, 22 (13), 2700-2708. https://doi.org/10.1002/adfm.201200249.

(7) Caruso, D.; Troisi, A. Long-Range Exciton Dissociation in Organic Solar Cells. Proceedings Of The National Academy Of Sciences Of The United States Of America 2012, 109 (34), 13498-13502. https://doi.org/10.1073/pnas.1206172109.

(8) Gregg, B. A. Entropy of Charge Separation in Organic Photovoltaic Cells: The Benefit of Higher Dimensionality. The Journal of Physical Chemistry Letters 2011, 2 (24), 3013-3015. https://doi.org/10.1021/jz2012403.

(9) Hood, S. N.; Kassal, I. Entropy and Disorder Enable Charge Separation in Organic Solar Cells. J. Phys. Chem. Lett. 2016, 7 (22), 4495-4500. https://doi.org/10.1021/acs.jpclett.6b02178.

(10) Athanasopoulos, S.; Tscheuschner, S.; Bässler, H.; Köhler, A. Efficient Charge Separation of Cold Charge-Transfer States in Organic Solar Cells Through Incoherent Hopping. J. Phys. Chem. Lett. 2017, 8 (9), 2093-2098. https://doi.org/10.1021/acs.jpclett.7b00595.

(11) Barbara, P. F.; Meyer, T. J.; Ratner, M. A. Contemporary Issues in Electron Transfer Research. The Journal of Physical Chemistry 1996, 100 (31), 13148-13168.

(12) MARCUS, R. A. On the Theory of Oxidation-Reduction Reactions Involving Electron Transfer. I. The Journal of Chemical Physics 1956, 24 (5), 966-978. https://doi.org/10.1063/1.1742723.

(13) Closs, G. L.; Miller, J. R. Intramolecular Long-Distance Electron Transfer in Organic Molecules. Science 1988, 240 (4851), 440-447. https://doi.org/10.1126/science.240.4851.440.

(14) Savoie, B. M.; Rao, A.; Bakulin, A. A.; Gélinas, S.; Movaghar, B.; Friend, R. H.; Marks, T. J.; Ratner, M. A. Unequal Partnership: Asymmetric Roles of Polymeric Donor and Fullerene Acceptor in Generating Free Charge. Journal Of The American Chemical Society 2014, 136 (7), 2876-2884. https://doi.org/10.1021/ja411859m.

(15) Coffey, D. C.; Larson, B. W.; Hains, A. W. An Optimal Driving Force for Converting Excitons into Free Carriers in Excitonic Solar Cells. The Journal of Physical Chemistry C 2012, 116 (16), 8916-8923. https://doi.org/10.1021/jp302275z.

(16) Pace, N. A.; Korovina, N. V.; Clikeman, T. T.; Holliday, S.; Granger, D. B.; Carroll, G. M.; Nanayakkara, S. U.; Anthony, J. E.; Mcculloch, I.; Strauss, S. H.; Boltalina, O. V.; Johnson, J. C.; Rumbles, G.; Reid, O. G. Slow Charge Transfer from Pentacene Triplet States at the Marcus Optimum. Nature Chemistry 2020, 12 (1), 63-70. https://doi.org/10.1038/s41557-019-0367-x.

(17) Ihly, R.; Mistry, K. S.; Ferguson, A. J.; Clikeman, T. T.; Larson, B. W.; Reid, O. G.; Boltalina, O. V.; Strauss, S. H.; Rumbles, G.; Blackburn, J. L. Tuning the Driving Force for Exciton Dissociation in Single-Walled Carbon Nanotube Heterojunctions. Nature Chemistry 2016, 8 (6), 603-609. https://doi.org/10.1038/nchem.2496.

(18) Placencia, D.; Wang, W.; Gantz, J.; Jenkins, J. L.; Armstrong, N. R. Highly Photoactive Titanyl Phthalocyanine Polymorphs as Textured Donor Layers in Organic Solar Cells. The Journal of Physical Chemistry C 2011, 115 (38), 18873-18884. https://doi.org/10.1021/jp2052085.

(19) Caplins, B. W.; Mullenbach, T. K.; Holmes, R. J.; Blank, D. A. Femtosecond to Nanosecond Excited State Dynamics of Vapor Deposited Copper Phthalocyanine Thin Films. Phys. Chem. Chem. Phys. 2016, 18 (16), 11454-11459. https://doi.org/10.1039/C6CP00958A.

(20) Park, J.; Reid, O. G.; Rumbles, G. Photoinduced Carrier Generation and Recombination Dynamics of 
a Trilayer Cascade Heterojunction Composed of Poly(3-Hexylthiophene), Titanyl Phthalocyanine, and C 60. Journal Of Physical Chemistry B 2014, 119 (24), 7729-7739.

https://doi.org/10.1021/acs.jpcb.5b00110.

(21) Dutton, G. J.; Robey, S. W. Exciton Dynamics at CuPc/C 60Interfaces: Energy Dependence of Exciton Dissociation. The Journal of Physical Chemistry C 2012, 116 (36), 19173-19181. https://doi.org/10.1021/jp305637r.

(22) Campoy-Quiles, M.; Müller, C.; Garriga, M.; Wang, E.; Inganäs, O.; Alonso, M. I. On the Complex Refractive Index of Polymer:Fullerene Photovoltaic Blends. Thin Solid Films 2014, 571, 371-376. https://doi.org/10.1016/j.tsf.2014.02.096.

(23) Moulé, A. J.; Meerholz, K. Interference Method for the Determination of the Complex Refractive Index of Thin Polymer Layers. Appl. Phys. Lett. 2007, 91 (6), 061901. https://doi.org/10.1063/1.2767232. Wu, J.; Luo, J.; Cernetic, N.; Chen, K.; Chiang, K.-S.; K.-Y. Jen, A. PCBM-Doped Electro-Optic Materials: Investigation of Dielectric, Optical and Electro-Optic Properties for Highly Efficient Poling. Journal of Materials Chemistry C 2016, 4 (43), 10286-10292. https://doi.org/10.1039/C6TC03932D. Buchaca-Domingo, E.; Vandewal, K.; Fei, Z.; Watkins, S. E.; Scholes, F. H.; Bannock, J. H.; de Mello J. C.; Richter, L. J.; DeLongchamp, D. M.; Amassian, A.; Heeney, M.; Salleo, A.; Stingelin, N. Direct Correlation of Charge Transfer Absorption with Molecular Donor:Acceptor Interfacial Area via Photothermal Deflection Spectroscopy. J. Am. Chem. Soc. 2015, 137 (16), 5256-5259. https://doi.org/10.1021/ja512410f.

(26) Benson-Smith, J. J.; Goris, L.; Vandewal, K.; Haenen, K.; Manca, J. V.; Vanderzande, D.; Bradley, D. D. C.; Nelson, J. Formation of a Ground-State Charge-Transfer Complex in Polyfluorene//[6,6]-Phenyl-C61 Butyric Acid Methyl Ester (PCBM) Blend Films and Its Role in the Function of Polymer/PCBM Solar Cells. Advanced Functional Materials 2007, 17 (3), 451-457. https://doi.org/10.1002/adfm.200600484.

(27) Albrecht, S.; Vandewal, K.; Tumbleston, J. R.; Fischer, F. S. U.; Douglas, J. D.; Fréchet, J. M. J.; Ludwigs, S.; Ade, H.; Salleo, A.; Neher, D. On the Efficiency of Charge Transfer State Splitting in Polymer:Fullerene Solar Cells. Advanced materials (Deerfield Beach, Fla.) 2014, 26 (16), 2533-2539. https://doi.org/10.1002/adma.201305283.

(28) Zhong, Y.; Causa, M.; Moore, G. J.; Krauspe, P.; Xiao, B.; Günther, F.; Kublitski, J.; Shivhare, R.; Benduhn, J.; BarOr, E.; Mukherjee, S.; Yallum, K. M.; Réhault, J.; Mannsfeld, S. C. B.; Neher, D.; Richter, L. J.; DeLongchamp, D. M.; Ortmann, F.; Vandewal, K.; Zhou, E.; Banerji, N. Sub-Picosecond Charge-Transfer at near-Zero Driving Force in Polymer:Non-Fullerene Acceptor Blends and Bilayers. Nature Communications 2020, 11, 833. https://doi.org/10.1038/s41467-020-14549-w.

(29) Reid, O. G.; Moore, D. T.; Li, Z.; Zhao, D.; Yan, Y.; Zhu, K.; Rumbles, G. Quantitative Analysis of Time-Resolved Microwave Conductivity Data. Journal of Physics D: Applied Physics 2017, 50 (49), 493002. https://doi.org/10.1088/1361-6463/aa9559.

(30) Savenije, T. J.; Ferguson, A. J.; Kopidakis, N.; Rumbles, G. Revealing the Dynamics of Charge Carriers in Polymer:Fullerene Blends Using Photoinduced Time-Resolved Microwave Conductivity. The Journal of Physical Chemistry C 2013, 117 (46), 24085-24103.

https://doi.org/10.1021/jp406706u.

(31) de Haas, M. P.; Warman, J. M.; Anthopoulos, T. D.; de Leeuw, D. M. The Mobility and Decay Kinetics of Charge Carriers in Pulse-lonized Microcrystalline PCBM Powder. 2006, 16 (17), 2274-2280. https://doi.org/10.1002/adfm.200500882.

(32) Ferguson, A. J.; Kopidakis, N.; Shaheen, S. E.; Rumbles, G. Dark Carriers, Trapping, and Activation Control of Carrier Recombination in Neat P3HT and P3HT: PCBM Blends. The Journal of Physical Chemistry C 2011, 115 (46), 23134-23148. https://doi.org/10.1021/jp208014v.

(33) Bao, D.; Millare, B.; Xia, W.; Steyer, B. G.; Gerasimenko, A. A.; Ferreira, A.; Contreras, A.; Vullev, V. I. Electrochemical Oxidation of Ferrocene: A Strong Dependence on the Concentration of the Supporting Electrolyte for Nonpolar Solvents. J. Phys. Chem. A 2009, 113 (7), 1259-1267. https://doi.org/10.1021/jp809105f.

(34) McNaught,A. D.; Wilkinson, A.; Chalk, S. J. IUPAC. Compendium of Chemical Terminology, 2nd Ed. 
(the "Gold Book"); Blackwell Scientific Publications: Oxford.

(35) Kawashima, E.; Fujii, M.; Yamashita, K. Entropy Promotes Charge Separation in Bulk Heterojunction Organic Photovoltaics. Journal Of Photochemistry And Photobiology A-Chemistry 2019, 382, 111875. https://doi.org/10.1016/j.jphotochem.2019.111875.

(36) Monahan, N. R.; Williams, K. W.; Kumar, B.; Nuckolls, C.; Zhu, X. Y. Direct Observation of Entropy-Driven Electron-Hole Pair Separation at an Organic Semiconductor Interface. Physical Review Letters 2015, 114 (24), 247003. https://doi.org/10.1103/PhysRevLett.114.247003. Xue, W.; Tang, Y.; Zhou, X.; Tang, Z.; Zhao, H.; Li, T.; Zhang, L.; Liu, S. (Frank); Zhao, C.; Ma, W.; Yan, $\mathrm{H}$. Identifying the Electrostatic and Entropy-Related Mechanisms for Charge-Transfer Exciton Dissociation at Doped Organic Heterojunctions. Advanced Functional Materials 2021, 31 (25), 2101892. https://doi.org/10.1002/adfm.202101892.

(38) Bakulin, A. A.; Rao, A.; Pavelyev, V. G.; van Loosdrecht, P. H. M.; Pshenichnikov, M. S.; Niedzialek, D.; Cornil, J.; Beljonne, D.; Friend, R. H. The Role of Driving Energy and Delocalized States for Charge Separation in Organic Semiconductors. Science 2012, 335 (6074), 1340-1344.

https://doi.org/10.1126/science.1217745.

(39) Pace, N. A.; Reid, O. G.; Rumbles, G. Delocalization Drives Free Charge Generation in Conjugated Polymer Films. ACS Energy Letters 2018, 3 (3), 735-741.

https://doi.org/10.1021/acsenergylett.8b00108.

(40) Feier, H. M.; Reid, O. G.; Pace, N. A.; Park, J.; Bergkamp, J. J.; Sellinger, A.; Gust, D.; Rumbles, G. Local Intermolecular Order Controls Photoinduced Charge Separation at Donor/Acceptor Interfaces in Organic Semiconductors. Advanced Energy Materials 2016, 6 (6), 1502176.

https://doi.org/10.1002/aenm.201502176.

(41) Barker, A. J.; Chen, K.; Hodgkiss, J. M. Distance Distributions of Photogenerated Charge Pairs in Organic Photovoltaic Cells. Journal Of The American Chemical Society 2014, 136 (34), 12018-12026. https://doi.org/10.1021/ja505380j.

(42) Gélinas, S.; Rao, A.; Kumar, A.; Smith, S. L.; Chin, A. W.; Clark, J.; van der Poll, T. S.; Bazan, G. C.; Friend, R. H. Ultrafast Long-Range Charge Separation in Organic Semiconductor Photovoltaic Diodes. Science 2014, 343 (6170), 512-516. https://doi.org/10.1126/science.1246249.

(43) Albinsson, B.; Mårtensson, J. Long-Range Electron and Excitation Energy Transfer in Donor-Bridge-Acceptor Systems. Journal of Photochemistry and Photobiology C: Photochemistry Reviews 2008, 9 (3), 138-155. https://doi.org/10.1016/j.jphotochemrev.2008.01.002.

(44) Ward, A. J.; Ruseckas, A.; Kareem, M. M.; Ebenhoch, B.; Serrano, L. A.; Al-Eid, M.; Fitzpatrick, B.; Rotello, V. M.; Cooke, G.; Samuel, I. D. W. The Impact of Driving Force on Electron Transfer Rates in Photovoltaic Donor-Acceptor Blends. Advanced materials (Deerfield Beach, Fla.) 2015, 27 (15), 2496-2500. https://doi.org/10.1002/adma.201405623.

(45) Nakano, K.; Chen, Y.; Xiao, B.; Han, W.; Huang, J.; Yoshida, H.; Zhou, E.; Tajima, K. Anatomy of the Energetic Driving Force for Charge Generation in Organic Solar Cells. Nature Communications 2019, 10, 2520. https://doi.org/10.1038/s41467-019-10434-3.

(46) Zhou, G.; Zhang, M.; Chen, Z.; Zhang, J.; Zhan, L.; Li, S.; Zhu, L.; Wang, Z.; Zhu, X.; Chen, H.; Wang, L.; Liu, F.; Zhu, H. Marcus Hole Transfer Governs Charge Generation and Device Operation in Nonfullerene Organic Solar Cells. ACS Energy Lett. 2021, 2971-2981. https://doi.org/10.1021/acsenergylett.1c01154.

(47) Karuthedath, S.; Gorenflot, J.; Firdaus, Y.; Chaturvedi, N.; De Castro, C. S. P.; Harrison, G. T.; Khan, J. I.; Markina, A.; Balawi, A. H.; Peña, T. A. D.; Liu, W.; Liang, R.-Z.; Sharma, A.; Paleti, S. H. K.; Zhang, W.; Lin, Y.; Alarousu, E.; Anjum, D. H.; Beaujuge, P. M.; De Wolf, S.; McCulloch, I.; Anthopoulos, T. D.; Baran, D.; Andrienko, D.; Laquai, F. Intrinsic Efficiency Limits in Low-Bandgap Non-Fullerene Acceptor Organic Solar Cells. Nat. Mater. 2021, 20 (3), 378-384. https://doi.org/10.1038/s41563-020-00835-x.

(48) Liu, J.; Chen, S.; Qian, D.; Gautam, B.; Yang, G.; Zhao, J.; Bergqvist, J.; Zhang, F.; Ma, W.; Ade, H.; Inganäs, O.; Gundogdu, K.; Gao, F.; Yan, H. Fast Charge Separation in a Non-Fullerene Organic Solar Cell with a Small Driving Force. Nat Energy 2016, 1, 16089. 
https://doi.org/10.1038/nenergy.2016.89.

(49) Zhou, J.; Findley, B. R.; Teslja, A.; Braun, C. L.; Sutin, N. Ion Pairs from Photoexcited, "Random" Electron Donors and Acceptors: Alkylbenzenes and Tetracyanoethylene. J. Phys. Chem. A 2000, 104 (49), 11512-11521. https://doi.org/10.1021/jp003005r.

(50) Ma, H.; Troisi, A. Direct Optical Generation of Long-Range Charge-Transfer States in Organic Photovoltaics. Advanced Materials 2014, 26 (35), 6163-6167. https://doi.org/10.1002/adma.201402294.

(51) Murthy, D. H. K.; Gao, M.; Vermeulen, M.; Siebbeles, L. D. A.; Savenije, T. J. Mechanism of Mobile Charge Carrier Generation in Blends of Conjugated Polymers and Fullerenes: Significance of Charge Delocalization and Excess Free Energy. The Journal of Physical Chemistry C 2012, 116 (16), 9214-9220. https://doi.org/10.1021/jp3007014.

(52) Grzegorczyk, W. J.; Savenije, T. J.; Dykstra, T. E.; Piris, J.; Schins, J. M.; Siebbeles, L. D. A. Temperature-Independent Charge Carrier Photogeneration in P3HT-PCBM Blends with Different Morphology. J. Phys. Chem. C 2010, 114 (11), 5182-5186. https://doi.org/10.1021/jp9119364.

(53) Gao, F.; Tress, W.; Wang, J.; Inganäs, O. Temperature Dependence of Charge Carrier Generation in Organic Photovoltaics. Physical Review Letters 2015, 114 (12), 128701. https://doi.org/10.1103/PhysRevLett.114.128701. 